\title{
$\mathbf{J}|\mathbf{A}| \mathbf{C} \mid \mathbf{S}$ \\ $\overline{\text { COMMUNICATIONS }}$
}

Published on Web 01/17/2002

\section{Differential Minor Groove Interactions between DNA Polymerase and Sugar Backbone of Primer and Template Strands}

Daniel Summerer and Andreas Marx*

Kekulé-Institut für Organische Chemie und Biochemie Universität Bonn, Gerhard-Domagk-Strasse 1, 53121 Bonn, Germany

Received October 5, 2001

DNA polymerases are the key enzymes for DNA synthesis involved in DNA replication, recombination, and repair. Structural insights of DNA polymerases and their mode of substrate binding and recognition were obtained from crystal structures of several DNA polymerases bound to their substrates. ${ }^{1}$ These studies as well as mechanistic investigations suggest that DNA polymerases make complex interactions with the primer template and nucleotide substrates during catalysis of DNA polymerization. ${ }^{2}$ Enzyme contacts with the primer template are manifold and reaching up to several nucleotide pairs beyond the catalytic center, leading in most cases to a severe bent of the DNA near the primer $3^{\prime}$-end. ${ }^{1 \mathrm{a}-\mathrm{e}, 2}$ These interactions primarily occur through the minor groove of the DNA duplex with the sugar phosphodiester backbone. ${ }^{1,2}$ To gain insights into the participation of these complex enzyme-DNA contacts in DNA polymerase function, we have developed new steric probes to investigate interactions through the minor groove acting on the sugar backbone. We report here on functional investigations leading to valuable new insights, which could be exploited for improved strategies based on the DNA polymerase reaction.

We expected that DNA modifications primarily cause altered kinetic properties of the enzyme through perturbation of native enzyme-substrate interactions at those positions where relevant DNA-enzyme contacts exist. To monitor steric constraints acting on the minor groove of DNA we applied 4'-alkyl modifications as steric probes which continually increase in steric bulk and point at the minor groove of double-stranded DNA (Figure 1) without significantly affecting hybridization properties. ${ }^{3-5}$ For this purpose alkyl groups should be ideally suited since potential effects on hydrogen-bonding patterns and conformations of the nucleotides are minimized. ${ }^{3}$ These properties significantly distinguish $4^{\prime}$-alkyl modifications from those applied in recent investigations of DNA polymerases, comprising probes with potential hydrogen-bond donors and acceptors or altered hydrogen-binding patterns. ${ }^{6}$ Furthermore, through application of the $4^{\prime}$-alkyl minor groove modifications in functional studies we should be able to monitor differential interactions acting on the primer and template strands.

We investigated the Klenow fragment (Kf) of Escherichia coli DNA polymerase I (exo-) since it is extensively used as a model for DNA polymerase mechanisms and function. ${ }^{2}$ To evaluate qualitatively contacts acting on the primer strand we performed primer extension studies employing site-specifically $4^{\prime}$-alkylated DNA as substrates (Figure 2). ${ }^{4}$ First, we tested the ability of Kf to perform primer extension from $4^{\prime}$-alkylated thymidine moieties at the 3 '-end of $25 \mathrm{nt}$ primers (position $+1 \mathrm{P}$ ) annealed to a $36 \mathrm{nt}$ template. ${ }^{4}$ Only negligible amounts of longer reaction products were detected, indicating that 4 '-alkylated sugar moieties strongly impede further DNA synthesis promoted by Kf (Figure 2A, lanes 1-5). However, at $+2 \mathrm{P}$ methylated and ethylated substrates were extended

* To whom correspondence should be addressed. E-mail: a.marx@uni-bonn.de

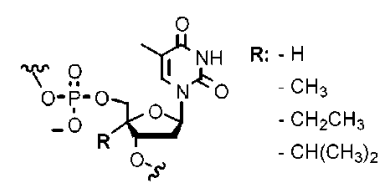

Figure 1. Nucleotide probes used in this study.

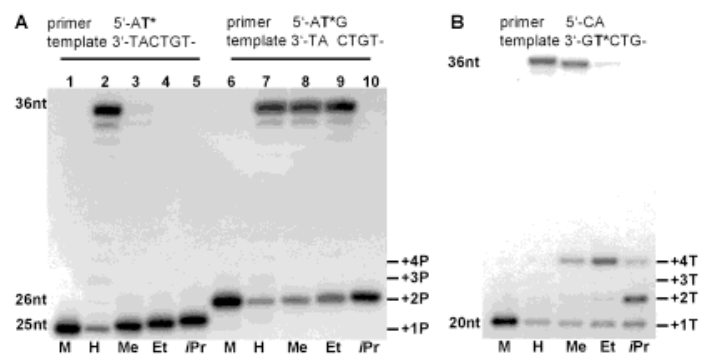

Figure 2. Effects of $4^{\prime}$-alkyl groups in the minor groove of DNA on Kfpromoted DNA synthesis. (A) Effects on primer. (B) Effects on template. Location of the modifications in the primer $(\mathrm{P})$-template $(\mathrm{T})$ are denoted $+\mathrm{n}, \mathrm{n}$ : nucleotide positions beyond the nucleotide binding pocket. $\mathrm{T}^{*}$ : thymidines bearing $4^{\prime}-\mathrm{H}$, methyl, ethyl, or isopropyl. M: marker. Reactions equally contained all dNTPs and Kf.

with significantly increased efficiency while isopropyl still prevents efficient extension (Figure 2A, lanes 6-10). To elucidate further remote minor groove contacts acting on the primer strand we synthesized DNA complexes comprising the 4'-alkylated moieties at positions $+1 \mathrm{P}$ to $+4 \mathrm{P} .{ }^{4}$ These $\mathrm{DNA}$ complexes serve as substrates for quantitative analysis of every single extension step employing single-nucleotide insertion assays under steady-state and single completed hit conditions as described recently. ${ }^{3,4,7}$ The results from the quantitative analysis showed that substitution of the $4^{\prime}$ hydrogen with a methyl group at $+1 \mathrm{P}$ cause a 2000 -fold reduction of nucleotide insertion efficiency. As the methyl modification moves further beyond the catalytic center such pronounced effects on the efficiency were no longer discernible. Bulkier ethyl and $i$-propyl groups interfere with DNA synthesis primarily at positions $+1 \mathrm{P}$ and $+2 \mathrm{P}$ while at $+3 \mathrm{P}$ and $+4 \mathrm{P}$ no significant effects were detected. The less efficient DNA synthesis observed at the two first nucleotides beyond the nucleotide binding pocket can be rationalized by most functionally important $\mathrm{Kf}$ interactions with the sugar moieties at $+1 \mathrm{P}$ and $+2 \mathrm{P}$. Interestingly, reduced extension efficiencies from sugar- or nucleobase-modified nucleotide analogues have been reported recently with $\mathrm{Kf}$ and other DNA polymerases, suggesting a more common phenomena. ${ }^{8}$

Next, we investigated $\mathrm{Kf}$ minor groove contacts with the template strand. Again we used a multiple nucleotide insertion assay to get a qualitative view of the action of $4^{\prime}$-alkylated template nucleotides on Kf bypass synthesis. To access contacts with the template strand within the DNA duplex we used a primer that places the modification at position +1 and performed nucleotide insertion employing all natural dNTPs (Figure 2B). In contrast to the results described 


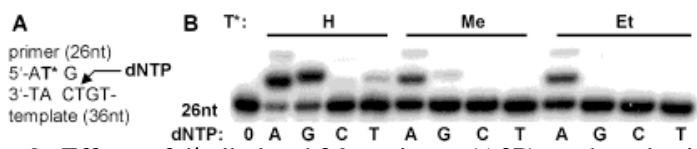

Figure 3. Effects of $4^{\prime}$-alkylated 26 nt primers $(+2 \mathrm{P})$ on the selectivity of Kf. (A) Partial sequences applied. ${ }^{4}$ (B) Results obtained after PAGE and autoradiography. All reactions contained equal concentrations of the respective single dNTP $(20 \mu \mathrm{M})$ and $\mathrm{Kf}(2 \mathrm{nM})$.

above the most prominent pausing site was observed at position $+4 \mathrm{~T}$ when a $4^{\prime}$-methylated template was employed. Increasing the size of the $4^{\prime}$-modification caused the accumulation of products at $+2 \mathrm{~T}$ and $+4 \mathrm{~T}$. Quantitative studies verified these observations and demonstrated that the effects on enzyme efficiency are more than 200 -fold at postion $+4 \mathrm{~T} .{ }^{4}$ Distal effects had been previously observed in Kf bypass synthesis of bulky DNA. ${ }^{6 c}$

Our results suggest differential interactions with primer and template strands through the protein. As shown by measured $T_{\mathrm{m}}$ values, ${ }^{4}{ }^{4}$-alkylation results in no significantly altered duplex hybridization. Thus, we attribute the observed effects mostly to steric interactions. No crystal structure of $\mathrm{Kf}$ bound to the DNA substrate in the DNA polymerization mode is available; thus, highly homologous Bst DNA polymerase I (Bst pol) is often used to correlate functional data to Kf structure. ${ }^{1 \mathrm{~d}, 2}$ The $B s t$ pol structure suggests close proximity of the sugar $4^{\prime}$-position to amino acid residues located in the turn of motif $\mathrm{C}$, namely Val828 (880 in Kf) and His829 (881) next to the essential Asp830 (882). ${ }^{1 \mathrm{~d}}$ Thus, modifications located close to the active site affect enzyme efficiencies most significantly. The Bst pol structure further indicates a set of contacts of a protein motif $\left(\beta\right.$-strand 8 in $\left.\mathrm{Kf}^{2 \mathrm{a}}\right)$, which stretches along the minor groove of the primer-template complex (610-626, 663-679 in Kf) and is in close proximity to the sugar moieties in $+2 \mathrm{P},+2 \mathrm{~T}$, $+4 \mathrm{~T}$. At these positions 4 '-modifications cause most pronounced distal effects on nucleotide insertion efficiency. These results indicate that $\mathrm{Kf}$ is able to translate distal perturbations at certain substrate positions to the active site, resulting in significantly diminished catalysis. ${ }^{6}$ These mechanisms may allow DNA polymerases to prevent sealing of damaged or altered nucleotides into the nascent DNA strand. Interestingly, on the basis of the structural data $+1 \mathrm{~T}$ modifications are expected to be located in close proximity to the backbone of Tyr714 (766), which corresponds to a residue in pol I family enzymes crucial for base flipping, which is believed to be involved in correct enzyme function. ${ }^{1 \mathrm{c}, \mathrm{d}}$ Methyl and ethyl substitutions at $+1 \mathrm{~T}$ exhibit only slight effects on nucleotide insertion.

The knowledge gained through this study should be useful for the design of new technologies based on the DNA polymerase reaction. In a first approach we investigated whether $4^{\prime}$-modified primer strands have any effects on the base-substitution fidelity of DNA synthesis. Higher DNA polymerase discrimination between canonical and noncanonical nucleotide insertion would be desirable for the development of improved methods for genome analysis, for example, the mini-sequencing approach. ${ }^{9}$ We speculated that addition of steric strain at $+2 \mathrm{P}$ through 4 '-alkyl modifications would cause less flexibility of the $3^{\prime}$-primer end and a decreased tolerance of geometrically altered nascent mispairs. Thus, we investigated single-nucleotide insertion by application of unmodified and $4^{\prime}$ alkylated primers, respectively (Figure 3).

Analysis of the reactions performed with unmodified primer revealed significant misinsertion (Figure 3). Employment of 4'methylated primer under identical conditions yielded only traces of misinsertion products, which were further diminished by the use of $4^{\prime}$-ethylated primer. Kinetic analysis of misinsertion compared to insertion revealed that $4^{\prime}$-modifications cause an increase of $K_{\mathrm{M}}$ accompanied by a remarkably reduced $V_{\max }$ in contrast to observations made with unmodified DNA substrates, where selectivity is primarily achieved by increase of $K_{\mathrm{M}} \cdot{ }^{4,10}$ These properties might be beneficial for improved DNA polymerase-based nucleotidedetection approaches. Together with our previous results, it appears that alkyl modifications at the sugar moieties decrease DNA polymerase substrate tolerance suggesting sugar backbone editing in Kf selectivity mechanisms. Furthermore, our results support the suggested model of geometrical selection as determinant of DNA polymerase selectivity. ${ }^{2}$

In conclusion, we describe new steric probes for the functional investigation of DNA polymerase interactions which target the sugar backbone in the minor groove of the primer template. This study shows that through application of the presented probes valuable insights into DNA polymerase function can be gained, which might be useful for the design of new DNA replication systems with improved desired properties.

Acknowledgment. We thank the DFG for funding. Professor M. Famulok is gratefully acknowledged for his continuing support and Dr. S. Verma for critical reading of the manuscript.

Supporting Information Available: Experimental details of synthesis, full DNA sequences, conditions applied in enzyme assays, kinetic data (PDF). This material is available free of charge via the Internet at http://pubs.acs.org.

\section{References}

(1) (a) Pelletier, H.; Sawaya, M. R.; Kumar, A.; Wilson, S. H. Kraut, J. Science 1994, 264, 1891-1903. (b) Doublié, S.; Tabor, S.; Long, A. M.; Richardson, C. C.; Ellenberger, T. Nature 1998, 391, 251-258. (c) Li, Y.; Korolev, S.; Waksman, G. EMBO J. 1998, 17, 7514-7525. (d) Kiefer, J. R.; Mao, C.; Braman, J. C.; Beese, L. S. Nature 1998, 391, 304-307. (e) Huang, H. F.; Chopra, R.; Verdine, G. L.; Harrison, S. C. Science 1998, 282, 1669-1675. (f) Franklin, M. C.; Wang, J.; Steitz, T. A. Cell 2001, 105, 657-667. (g) Ling, H.; Boudsocq, F.; Woodgate, R.; Yang, W. Cell 2001, 107, 91-102.

(2) Recent reviews: (a) Joyce, C. M.; Steitz, T. A. Annu. Rev. Biochem. 1994 63, 777-822. (b) Kunkel, T. A.; Bebenek, K. Annu. Rev. Biochem. 2000, 69, 497-529. (c) Kool, E. T.; Morales, J. C.; Guckian, K. M. Angew. Chem., Int. Ed. 2000, 39, 991-1009. (d) Patel, P. H.; Suzuki, M.; Adman, E.; Shinkai, A.; Loeb, L. A. J. Mol. Biol. 2001, 308, 823-837. (e) Patel, P. H.; Loeb, L. A. Nat. Struct. Biol. 2001, 8, 656-659.

(3) Summerer, D.; Marx, A. Angew. Chem., Int. Ed. 2001, 40, 3696-3698.

(4) Details of the synthesis of modified oligonucleotides, full sequences of DNA substrates, selected $T_{\mathrm{m}}$ data, and kinetic data of quantitative DNA polymerase assays are provided in the Supporting Information.

(5) Saenger, W. In Principles of Nucleic Acid Structure; Cantor, C., Ed.; Springer, New York, 1993

(6) (a) Charry, P.; Harris, C. M. Harris, T. M. Lloyd, R. S. J. Biol. Chem. 1997, 272, 5805-5813. (b) Forgacs, E.; Latham, G.; Beard, W. A.; Prasad, R.; Bebenek, K.; Kunkel, T. A.; Wilson, S. H.; Lloyd, R. S. J. Biol. Chem. 1997, 272, 8525-8530. (c) Hess, M. T.; Schwitter, U.; Petretta, M.; Giese, B.; Naegli, H. Biochemistry 1997, 36, 2332-2337. (d) Marx, A.; Spichty, M.; Amacker, M.; Schwitter, U.; Hübscher, U.; Bickle, T. A.; Maga, G.; Giese, B. Chem. Biol. 1998, 6, 111-116. (e) Suo, Z.; Lippard, S. J. Johnson; K. A. Biochemistry 1999, 38, 715-726. (f) Latham, G. J.; Forgacs, E.; Beard, W. A.; Prasad, R.; Bebenek, K.; Kunkel, T. A.; Wilson, S. H.; Lloyd, R. S. J. Biol. Chem. 2000, 275, 15025-15033.

(7) Creighton, S.; Bloom, L. B.; Goodman, M. F. Methods Enzymol. 1995, $262,232-256$

(8) (a) Marx, A.; MacWilliams, M. P.; Bickle, T. A.; Schwitter, U.; Giese, B. J. Am. Chem. Soc. 1997, 119, 1131-1132. (b) Matray, T. J.; Kool, E. T. Nature 1999, 399, 704-708. (c) Kool, E. T.; Morales, J. C. J. Am. Chem. Soc. 1999, 121, 2323-2324. (d) Kool, E. T.; Morales, J. C. J. Am. Chem. Soc. 2000, 122, 1001-1007. (e) Ogawa, A. K.; Wu, Y.; McMinn, D. L.; Liu, J.; Schultz, P. G.; Romesberg, F. E. J. Am. Chem. Soc. 2000, 122, 3274-3287. (f) Wu, Y.; Ogawa, A. K.; Berger, M.; McMinn, D. L.; Liu, J.; Schultz, P. G.; Romesberg, F. E. J. Am. Chem. Soc. 2000, 122, 3274-3287. (g) Vastmans, K.; Pochet, S.; Peys, A.; Kerremans, L.; Van Aerschot, A.; Hendrix, C.; Marlière, P.; Herdewijn, P. Biochemistry 2000, 39, 12757-12765. (h) Vastmans, K.; Froeyen, M.; Kerremans, L.; Pochet, S.; Herdewijn, P. Nucleic Acids Res. 2001, 29, 3154-3163. (i) Tae, E. L.; Wu; Y.; Xia, G.; Schultz, P. G.; Romesberg, F. E. J. Am. Chem. Soc. 2001, $123,7439-7440$

(9) (a) Syvänen, A.-C. Hum. Mut. 1999,13, 1-10. (b) Pirrung, M. C.; Connors, R. V.; Odenbaugh, A. L.; Montage-Smith, M. P.; Walcott, N.; Tollett, J. J. J. Am. Chem. Soc. 2000, 122, 1873-1882.

(10) Goodman, M. F.; Creighton, S.; Bloom, L. B.; Petruska, J. Crit. Rev. Biochem. Mol. Biol. 1993, 28, 83-126. JA017244J 